$$
\triangle N F-9 \approx C 6 C=-7.5
$$

Presented at the 1992 Applied Superconductivit: Conference

Chicago, IL dugust 23-28, 1992

$\mathrm{BNL}-48152$

\title{
LOCATING THE MIAGNETIC CENTER OF THE SSC CDM USING A TEMPORARY QUADRUPOLE FIELD
}

\author{
C. R. Gibson, D. W. Bliss and R. E. Simon \\ General Dynamics Space Systems Division \\ San Diego, CA. $92186-5990$
}

A. K. Jain and P. Wanderer

Brookhaven National Laboratory

Upton, NY. 11973
BNL --48152

DE93 008058

\begin{abstract}
The SSC dipole magnets must be very accurately aligned for the accelerator to work correculy. To align the magnets one must accurately know the center of the magnetic field. However, locating the center is difficult because of the extreme uniformity of the dipole field. A technique has been developed to determine the magnetic center of the SSC dipole magnet under ambient test conditions. This technique involves flowing current in the upper half of the coils in the direction opposite to the current in the lower half. This is done using a temporary lead connected to the splice joining the two coil halves and two matched power supplies. The resulting field is primarily skew quadrupole and has high field gradients which allow the center to be accurately located. The viability of this method has been verified by analysis using Taylor series expansion of the resulting magnetic field. Analysis has also verified that off-the-shelf power supplies have low enough mismatch to be used in this technique. The analytical results have been verified through the test of a shor dipole magnet at the Brookhaven National Laboratory.
\end{abstract}

\section{INTRODUCTION}

In order for the Superconducting Super Collider (SSC) to meet its physics requirements, the magnetic center of each Collider Dipole Magnet (CDM) must be accurately aligned. The two most commonly used methods to find the center of a magnet are geometric and magnetic. The geometric method is based on the assumption that the magnetic center of the magnet is coincident with the geometric center. This is a good assumption for the CDM because the coils are extremely uniform and are two-dimensional in shape. Difficulty arises however after the magnet is assembled since the coils are not directly accessible. After assembly, one can use the beam wbe as a reference but this introduces additional errors due to the tolerances of the tube and its locating method.

The magnetic method locates the center of the dipole field directly. This method uses a magnetic field measuring device, a one meter long rotating coil commonly called a "mole", in concert with feeddown analysis. Feeddown analysis is a technique which uses both high order allowed harmonics and lower order unallowed harmonics to analytically locate the magnetic center. While this technique generally gives consistent results, problems may arise if the higher order terms are not measured to sufficient accuracy or when the measuring coil radius small compared to the magnet bore radius. Both of these problems occur in the CDM because of

Manuscript received August 24, 1992 the extreme purity of the magnetic field and the fact that the moles have an average coil radius of $12 \mathrm{~mm}$ while the magnet bore radius is $25 \mathrm{~mm}$.

An altemate technique has been developed which allows the use of direct magnetic centering while avoiding the problems associated with using feeddown on a dipole magnet. This technique involves flowing current in the upper half of the coils in the direction opposite to the current in the lower half. The resulting field is predominantly skew quadrupole, as shown in Figure 1, and has high field gradients which allow the center to be accurately located using a mole and feeddown analysis. It should be noted that this is not a true quadrupole, since symmetry about $45^{\circ}$ and $135^{\circ}$ axes is not satisfied, and substantial amounts of higher order terms are present. We shall, however, refer to this technique as the "skew quadrupole centering technique".

Reversing the current direction in the lower half of the magnet is done using a temporary lead connected to the splice joining the two coil halves. Locating the magnetic center of the CDM is performed warm during fabrication using approximately $10 \mathrm{~A}$, and hence only a small copper temporary lead is required. The two coil halves are each connected to a matched power supply to ensure that each half has identical currents. Analysis presented in this paper shows that off-theshelf power supplies have low enough mismatch to be used in this technique.

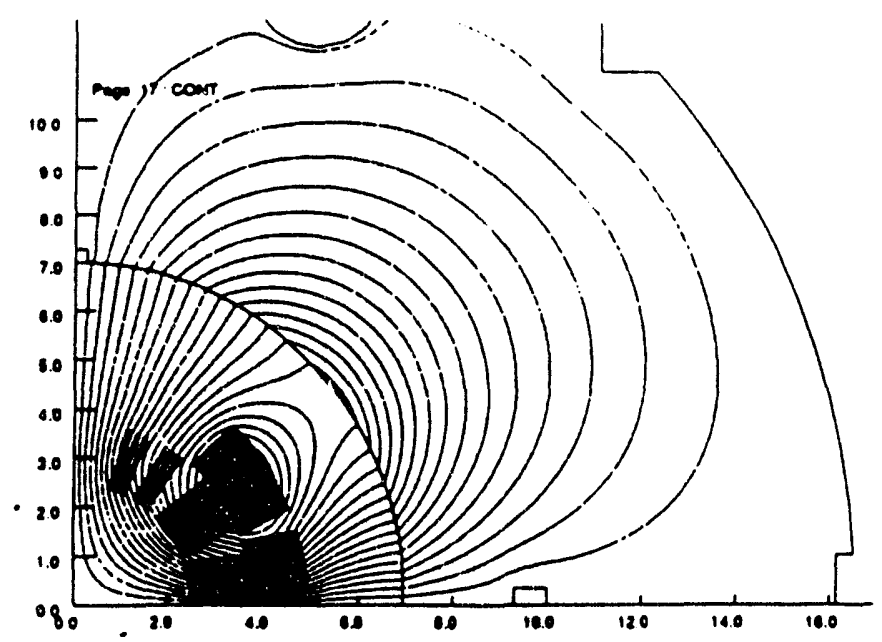

Fig. $190^{\circ}$ model of the CDM connected in the skew quadrupole configuration.

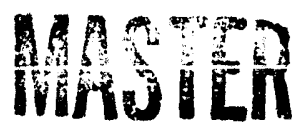




\section{DISCLAIMER}

This report was prepared as an account of work sponsored by an agency of the United States Government. Neither the United States Government nor any agency thereof, nor any of their employees, makes any warranty, express or implied, or assumes any legal liability or responsibility for the accuracy, completeness, or usefulness of any information, apparatus, product, or process disclosed, or represents that its use would not infringe privately owned rights. Reference herein to any specific commercial product, process, or service by trade name, trademark, manufacturer, or otherwise does not necessarily constitute or imply its endorsement, recommendation, or favoring by the United States Government or any agency thereof. The views and opinions of authors expressed herein do not necessarily state or reflect those of the United States Government or any agency thereof. 


\section{FEEDDOWN ANALYSIS}

\section{A. Feeddown}

Feeddown occurs when the measuring coil center is not coincident with the magnet center. The measured values of magnetic field, when broken down into harmonic coefficients $b_{n}$ and $a_{n}$, include the effects of feeddown from higher order terms.

The ability to find the magnetic center using feeddown from high order harmonics is limited by the accuracy of the harmonic measurements. With the re-optimized CDM trosssection, the high order harmonics are extremely small. Using a required magnetic field center location accuracy of $110 \mu \mathrm{m}$, the required measurement accuracy of $b_{9}$ and $b_{10}$ is better than 0.0014 units in the dipole configuration. The mole may be able to measure the field this accurately. Currently, the accuracy of the mole is believed to be better than 0.01 units; how much better is unknown. In addition, due to the difficulty in calibrating the mole, its accuracy may remain unknown throughout the SSC program. Consequently, use of the skew quadrupole centering scheme has been investigated as an alternative technique.

\section{B. Feeddown Definition}

The purpose of the following analysis is to determine the required tolerance for mole harmonics measurement. The magnetic fields can be represented either by a Fourier series or Taylor series expansion. For feeddown analysis it is convenient to use the Taylor series representation.

$$
\begin{aligned}
& B=B_{x}+i B_{y}=\sum_{n=0}^{\infty}\left(D_{n}\right)\left(z^{*}\right)^{n} ; \\
& z=x+i y
\end{aligned}
$$

The feeddown effect is produced by measuring the magnetic field with an origin that has an offset from the magnetic center. For this analysis the offset is a complex number. Replacing $z$ with $(z+\delta)$, where $z^{\prime}$ is the measurement variable and $\delta$ is the offset from the magnetic center, the series can be transformed to show the harmonic produced by measuring off center.

$$
\begin{aligned}
& B=\sum_{n=0}^{\infty}\left(D_{n}\right)\left(\left[z^{*}+\delta\right]^{*}\right)^{n} ; z=z^{*}+\delta \\
& B=\sum_{n=0}^{\infty}\left(D_{n}\right)\left[\left(z^{*}\right)^{*}\right]^{n}\left[1+n \frac{\delta^{*}}{z^{*}}\right] ;
\end{aligned}
$$

taking the first two terms of the binomial expansion

Since the $\delta / z^{\prime}$ multiplies the $\left(z^{\prime}\right)^{n}$ term, it appears to produce a $z^{\prime}(n-1)$ component. This is the method in which feeddown is produced.

$$
B=\sum_{n=0}^{\infty}\left[\left(z^{\prime}\right)^{*}\right]^{n}\left[\left(D_{n}\right)+(n+1) \delta *\left(D_{n}+1\right)\right]
$$

If $D_{\mathbf{n}}^{\prime}$ is the measured harmonic,

$$
\begin{aligned}
& B=\sum_{n=0}^{\infty}\left[\left(z^{\prime}\right)^{*}\right]^{n}\left(D_{n}^{\prime}\right) \\
& D_{n}^{\prime}=\underset{\substack{\left(D_{n}\right)+(n+1) \\
\text { comparing coefficients }}}{\left(D_{n+1}\right):}
\end{aligned}
$$

For the SSC dipole magnets odd coefficients, when considered from the magnetic center, are produced by coil asymmetries. When considering higher order harmonics, odd harmonics should be very small. They can therefore be ignored. Feeddown to $D_{n+1}$ is small for two reasons. First, $D_{n+2}$ is very small because it is odd harmonic for odd $n$. It is therefore a weak feeddown source. Second, feeddown from $D_{n+3}$ and higher terms are reduced by $(\delta / 2)^{k-(n+1)}$, where $k$ is $n+3$ or larger; they can be ignored if $\delta / 2$ is small. This is same reason used for truncating the binomial expansion in equation (4). Consequently, measured $\mathbf{D}_{\mathbf{n}}^{\prime}+1$ is approximately equal to $\mathbf{D}_{\mathbf{n}_{+}}$. Using this information the origin displacement can be found.

$$
\delta=\frac{1}{n+1} \frac{\left(D_{n}^{\prime}\right)^{*}}{\left(D_{n}^{\prime}+1\right)^{*}}
$$

\section{Requirements on Measwrement Accuracy}

The CDM alignment plan requires the magnetic center to be located to within $110 \mu \mathrm{m}$. This tolerance sets the accuracy of measurements required of the harmonics. Since $\Delta \delta$ is small, derivatives can be used to determine the sensitivity to $D$ variation. Since the requirement on $\delta$ is isotropic, only magnitudes need 10 be considered.

Since $D_{n}^{\prime}$ and $D_{n+1}^{\prime}$ are close harmonics measurements, one would expect that measurement accuracy for both would be similar. Assuming this to be exactly true, a tolerance can be determined. Determining the sensitivity to measurement from equation (8) and adding in quadranure.

$$
\begin{aligned}
& \Delta \delta= \Delta D \frac{1}{n+1} \sqrt{\frac{D_{n}^{-2}}{\left(D_{n+1}^{\prime}\right)^{4}}+\frac{1}{\left(D_{n+1}^{\prime}\right)^{2}}} ; \\
& \Delta D \text { is the harmonic measurement accuracy }
\end{aligned}
$$

Since $D_{n}$ is a feeddown produced term, it can be replaced with its feeddown source function, from equation (8). By substituting this function in for $D_{n}^{\prime}, \Delta D$ can be found. Substituting and using $D_{n+1}^{\prime} \approx D_{n+1}$

$$
\Delta D: \frac{\Delta \delta(n+1)\left(D_{n+1}^{\prime}\right)}{\sqrt{[\delta(n+1)]^{2}+1}}
$$


In the CDM cross-section selected for the first seven prototype magnets, the value of $b_{10}$ is large compared to other harmonics in its neighborhood. Consequently, it is a logical choice for a feeddown source. Assuming a value of 0.018 units for the 22-pole term, and a offset of $\delta=1 \mathrm{~mm}$, we get the required accuracy of measurement of 0.0014 units for high order terms in order to achieve $\Delta \delta=110 \mu \mathrm{m}$. It is important to perform the previous calculation with lengths in terms of centimeters since centimeters is implicit in "unit". The term "unit" indicates 10,000 times the field gradient in terms of Tesla per centimeter to the $n^{\text {th }}$ power normalized by the central ñeld in terms of Tesla.

\section{Power Supply Requirements}

While locating the magnetic center, two matched power supplies, set up as master and slave, are used to ensure that equal currents flow in the top and bottom coil halves. Commercial power supplies are available off-the-shelf with a maximum mismatch specification of $0.1 \%$. An analysis has been performed to determine if these commercial power supplies meet the requirements for the skew quadrupole centering technique.

When a current mismatch is introduced, changes in the field harmonic values result which translate into errors in the offset calculations. The change in the calculated offset can be determined from the change in the harmonic. An analysis has been performed using the computer code PE2D and a $180^{\circ}$ model of the $50 \mathrm{~mm}$ aperature CDM, similar to the one shown in Figure 1. Current values were scaled to $10.00 \mathrm{~A}$ (10.01 $\mathrm{A}$ in the upper coils of the mismatch configuration) in the simulations. The results of the simulation are shown in Table 1 which lists the magnitudes of the lower order harmonic coefficients, $D_{0}, D_{2}$ and $D_{4}$, for the matched and unmatched current situations. Higher order harmonics are so small that feeddown from them can be neglected.

Table 1. Harmonics Coefficient Magnitudes (G/ $\mathrm{cm}^{\mathrm{n}}$ )

\begin{tabular}{|c|c|c|c|c|}
\hline$n$ & $\begin{array}{c}\left|D_{n}\right| \\
\text { (matched) }\end{array}$ & $\begin{array}{c}\left|D_{n+1}\right| \\
\text { (matched) }\end{array}$ & $\begin{array}{c}\left|D_{n}\right| \\
\text { (unmatched) }\end{array}$ & $\begin{array}{c}\left|D_{n+1}\right| \\
\text { (unmauched) }\end{array}$ \\
\hline 0 & 0.00020 & 20.1034 & 0.05188 & 2.1134 \\
2 & $1.93 \mathrm{E}-5$ & 0.62620 & $2.06 \mathrm{E}-5$ & 0.62650 \\
4 & $2.13 \mathrm{E}-5$ & 0.03797 & $2.11 \mathrm{E}-5$ & 0.03799 \\
\hline
\end{tabular}

The largest change in calculated offset is $25.7 \mu \mathrm{m}$ stemming from the change in the $D_{0}$ harmonic. None of the other harmonic changes affect the calculated offset by more than $0.015 \mu \mathrm{m}$. Therefore, a $0.1 \%$ maximum current mismatch requirement is not excessively stringent and current mismatch should not be a limiting factor in using the skew quadrupole centering technique.

\section{TEST RESULTS}

The feasibility of using the skew quadrupole centering method was investigated experimentally in a shor magnet DS0204. This is a shor SSC dipole magnet with coils of $40 \mathrm{~mm}$ inner diameter. In normal operation of the magnet. there is an external splice between the upper and lower coils.
For this experiment, this splice was taken apart. This facilitated measurements in either the usual dipole mode, or the skew quadrupole mode, by using suitable jumper cables and a single power supply. This also ensured that the currents in the upper and the lower halves of the magnet are the same in both the modes. In an actual application for the collider magnets, however, two independent power supplies will be needed to power the upper and the lower coils in the skew quadrupole mode.

Field measurements were made in the magnet DSO204 in both the dipole and the skew quadrupole configurations at a current of $10 \mathrm{~A}$. The dipole field at this current is about 100 Gauss in the dipole configuration. In the case of the skew quadrupole configuration, simple analytical estimates can be oblained by assuming a $|\cos \theta|$ dependence of the magnitude of current density. Such an estimate gives field strengths of 25.9 and 1.3 Gauss at $1.0 \mathrm{~cm}$ radius for the skew quadrupole and the skew octupole respectively in this magnet at $10 \mathrm{~A}$. These are substantial field levels, and can be measured very precisely. The dipole, sextupole, etc., terms are unallowed, and are expected to be negligible in comparison. However, there may be a significant permanent dipole field from the iron yoke. The data can be corrected to subtract this permanent field by making measurements at both $+10 \mathrm{~A}$ and $-10 \mathrm{~A}$. The magnet was taken through three $\mathrm{AC}$ cycles of $\pm 10 \mathrm{~A}$ in the beginning of each run, before taking data, to minimize the residual fields. Seven sets of data, each consisting of $a+10 \mathrm{~A}$ and a -10 A measurement, were taken for each configuration.

\section{A. Experimental Resulis}

Figure 2 shows the typical harmonic fields contributed by the coil (filled boxes) and the iron yoke (crosses) in one of the measurements in the skew quadrupole configuration. The separate contributions were calculated by using the $+10 \mathrm{~A}$ and the - $10 \mathrm{~A}$ data. It is seen that the residual dipole field can be even greater than the dipole field (due to feeddown) from the coil. Thus, quadrupole feeddown can not be used for centering. unless the iron effect is subtracted out from the data. As a check on sensitivity, the histogram in Figure 2 depicts the detection limits of various harmonic fields assuming a conservative voltage sensitivity of $0.1 \mu \mathrm{V}$. It is seen that all the harmonic field from the coil, including the unallowed terms up to the 20-pole, are well above the detection limit. Also, the measured quadrupole and octupole strengths at $1.0 \mathrm{~cm}$ radius were 25.6 and 1.2 Gauss respectively, in very good agreement with the approximate analytical calculations.

\section{B. Censering Paramelers}

In the case of the usual dipole configuration, the centering parameters $\left(r_{0}, \omega\right)$ describing the vector $\delta$ in eq. (8) were obtained by minimizing feeddown from the allowed 22-pole and 18-pole terms to the unallowed 20-pole and 16-pole respectively. The resulting $r_{0}$ values are shown in Figure 3 by crosses for all the 14 records in the run (no averaging of $+10 \mathrm{~A}$ and $-10 \mathrm{~A}$ data was done in this case). The average $r_{0}$ was $0.497 \mathrm{~mm}$ and the standard deviation (s.d.) from the 14 records was $143 \mu \mathrm{m}$. The $\pm 1 \sigma$ range is denoted by the dashed lines in Figure 3. The scatter of the ro values is quite large in 


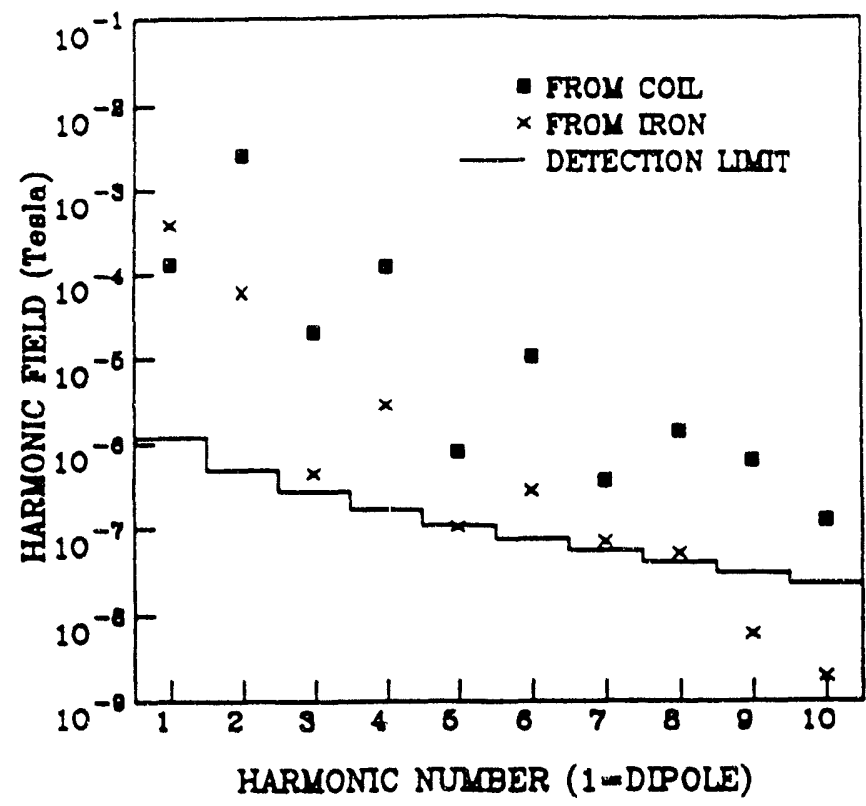

Fig. 2 Detection limits of various harmonic field numbers assuming a voltage sensitivity of $0.1 \mu \mathrm{V}$.

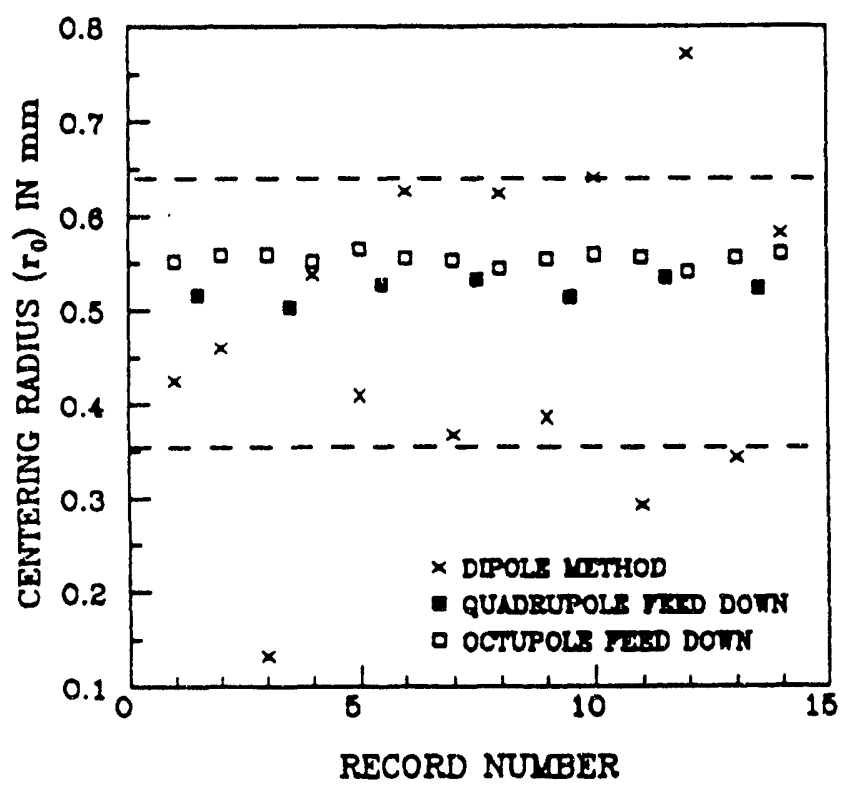

Fig. 3 Centering radius ( $r 0$ in DSO204 for both dipole and skew quadrupole centering methods.

this case. The angle parameter $\omega$ shows a similar scatter and the mean value is 327.6 degrees with a s.d. of 26.1 degrees. If :he $+10 \mathrm{~A}$ and $-10 \mathrm{~A}$ data are averaged to subtract the residual fields, the scatter can be reduced to a s.d. of $60 \mu \mathrm{m}$ in this case.

In the case of the skew quadrupole configuration, $(r 0, \omega)$ were obtained by requiring no feeddown from the quadrupole term. The 14 records of the run give only 7 values of $(r 0, \omega)$ in this case, since the data from $+10 \mathrm{~A}$ and $-10 \mathrm{~A}$ must be averaged in this case to get rid of the residual iron fields. The scatter in this case is remarkably small, and the mean $r_{0}$ is $0.521 \mathrm{~mm}$ with a s.d. of $11 \mu \mathrm{m}$. The mean value of $\omega$ is 314.2 degrees with a s.d. of 1.1 degrees. These values are consistent with those calculated from the usual method of high order terms feeddown in the dipole configuration. The improved statistics in the new method is a direct result of working at field levels which are several orders of magnitude higher than typical detection limits (see Figure 2).

Although excellent results are obtained by the quadrupole feeddown method, the accuracy may still be compromised by the fact that the residual dipole field is higher than that from the coil, making accurate subtraction difficult. A close look at Figure 2 suggests that the best choice in this case would be to use feeddown from the octupole $(n=4)$ term. This is because for both $n=4$ and $n=3$, the field levels are still much higher than the detection limits, and the residual fields from the iron are negligible compared to the fields from the coil. Also, it is not essential (although still preferable) to average the $+10 \mathrm{~A}$ and the $-10 \mathrm{~A}$ data. The centering parameter $r_{0}$ obtained by octupole feeddown is also shown in Figure 3 by open boxes. The s.d. in this case is only $6 \mu \mathrm{m}$, even though no subtraction of residual fields was performed.

The skew quadrupole method of centering has also been tested with two independent power supplies for the upper and the lower coils in measurements at $15 \mathrm{~K}$ and $4.35 \mathrm{~K}$ with equally consistent results. [1]

\section{SUMMARY AND CONCLUSIONS}

The skew quadrupole centering technique has been developed 10 accurately locate the center of a dipole magnet during fabrication. Tests results on magnet DS0204 have shown that this method can provide extremely reliable centering parameters, which can then be used to analyze field data in the usual dipole configuration.

\section{ACKNOWLEDGMENTS}

The work described herein is being accomplished under contract to the Universities Research Association in support of the Superconducting Super Collider project for the U.S. Deparment of Energy.

\section{REFERENCES}

[1] R. Howard, A. K. Jain and P. Wanderer, "Centering Parameters in DRS8", BNL Magnet Division Note 434. 11 (RHIC-MD-145), April 14, 1992. 


